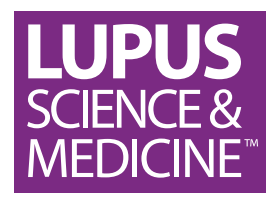

\title{
Plasma levels of galectin-3-binding protein reflect type I interferon activity and are increased in patients with systemic lupus erythematosus
}

\author{
Christoffer T Nielsen, ${ }^{1,2}$ Christian Lood, ${ }^{3}$ Ole Østergaard, ${ }^{1}$ Line V Iversen, ${ }^{1,4}$
} Anne Voss, ${ }^{5}$ Anders Bengtsson, ${ }^{3}$ Søren Jacobsen, ${ }^{2}$ Niels $\mathrm{H} \mathrm{H}$ Heegaard ${ }^{1}$

To cite: Nielsen CT, Lood C, Østergaard 0, et al. Plasma levels of galectin-3-binding protein reflect type I interferon activity and are increased in patients with systemic lupus erythematosus. Lupus Science \& Medicine 2014;1: e000026. doi:10.1136/lupus2014-000026

Received 31 January 2014 Revised 6 August 2014 Accepted 16 October 2014

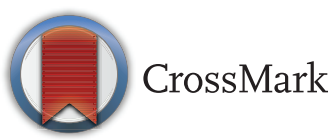

For numbered affiliations see end of article.

Correspondence to Dr Christoffer Tandrup Nielsen; christoffertandrupnielsen@ gmail.com

\section{ABSTRACT}

Objective: Simple measures of type I interferon (IFN) activity constitute highly attractive biomarkers in systemic lupus erythematosus (SLE). We explore galectin-3-binding protein (G3BP) as a novel measure of type I IFN activity and serum/plasma biomarker in large independent cohorts of patients with SLE and controls.

Methods: Serum and plasma G3BP concentrations were quantified using ELISA. Type I IFN activity was assessed by Mx1 reporter gene expression assays and correlated to serum G3BP concentrations (SLE-IFN- $\alpha$, $\mathrm{n}=26$ and healthy controls $(\mathrm{HCs}), \mathrm{n}=10$ ). Plasma G3BP concentrations in the SLE-Denmark (DK) $(n=70)$ and SLE-Sweden (SE) $(n=68)$ cohorts were compared with the HC-DK ( $n=47)$ and HC-SE $(n=50)$ cohorts and patients with systemic sclerosis $(n=111)$. In 15 patients with SLE, serum G3BP in consecutive samples was correlated to disease activity. Correlation analysis between G3BP, clinical parameters including disease activity in the four SLE cohorts was performed.

Results: G3BP concentrations correlated significantly with the IFN- $\alpha$ reporter gene assay $(r=0.56, p=0.0005)$ and with IFN- $\alpha$ gene expression scores $(r=0.54$, $p=0.0002$ ). Plasma concentrations were significantly increased in the SLE-DK and SLE-SE cohorts compared with $\mathrm{HCs}$ and patients with systemic sclerosis $(p<0.0001$ and $p=0.0009)$. G3BP concentrations correlated with disease activity measures in the SLE-DKand SLE-IFN- $\alpha$ cohorts $(p=0.0004$ and $p=0.05$ ) but not in the SLE-SE cohort $(p=0.98)$. Markedly temporal variation was observed in G3BP levels in the consecutive SLE-samples and was significantly associated with changes in disease activity ( $r=0.44$, $\mathrm{p}=0.014$ ).

Conclusions: G3BP plasma levels reflect type I IFN activity and are increased in SLE. Associations with disease activity or clinical manifestations are uncertain. This study highlights G3BP as a convenient measure of type I IFN-dependent gene activation.

\section{INTRODUCTION}

Systemic lupus erythematosus (SLE) is a severe systemic autoimmune disease characterised by the loss of immunological

\section{KEY MESSAGES}

Soluble galectin-3-binding protein is a convenient biomarker of type I interferons.

- Soluble galectin-3-binding protein is elevated in patients with SLE and associated with high disease activity, while the clinical and serological associations are uncertain.

tolerance against nuclear antigens. The disease course is unpredictable with alternating periods of flares and remissions. The clinical and paraclinical tools to assess disease activity and predict the disease course are often inadequate and identification of new and easily accessible biomarkers is highly needed in SLE.

A sustained production of type I interferons (IFNs), defective clearance of dying cells, formation and deposition of immune complexes (ICs), dysregulation of antigenpresenting $\mathrm{T}$ cells and $\mathrm{B}$ cells and possibly the presence of an abnormal circulating population of microparticles (MPs) are key components in the development of antinuclear autoimmunity in SLE. ${ }^{2}$ The ongoing type I IFN production in SLE has been known for decades, and patients with SLE often display an overexpression of IFN-inducible genes in peripheral blood mononuclear cells (PBMCs), the so-called type I IFN gene signature. ${ }^{3} 4$ Type I IFNs have been linked to autoantibody profiles, particularly to antibodies against ribonucleoproteins, to specific disease manifestations, such as skin rash, fever, haematological abnormalities, renal and CNS-involvement, complement activation, high disease activity and prediction of flares. ${ }^{13-10}$ These observations have triggered current clinical drug trials targeting type I IFNs in SLE. ${ }^{11}$ Thus IFN- $\alpha$ and indirect measures of type I IFN 
activity such as the levels of IFN- $\alpha$ regulated proteins or profiling of the transcripts of IFN-inducible genes are of interest as biomarkers in SLE. Often composite scores of IFN-induced gene transcripts have been employed where the combination ensures the specificity and sensitivity for IFN- $\alpha$. Assessment of type I IFN-activity at the protein level, however, may be more accessible and convenient and less sensitive to leucocyte levels. Recently, IFN $\gamma$-inducible protein 10 (IP-10, CXL10), monocyte chemotactic protein-1 (MCP-1, CCL2), chemokine ligand 19, or sialic acid-binding Ig-like lectin-1 on monocytes (SIGLEC-1) were shown to be less affected by fluctuations in leucocyte levels and more sensitive than IFN- $\alpha$ and IFN-inducible gene scores in assessing high disease activity. On the other hand, the stronger association of these protein markers with disease activity may be partially attributable to other non-IFN- $\alpha$ inflammatory cytokines. $^{7810}$

We here focus on galectin-3-binding protein (G3BP, 90K/Mac-2-binding protein) in solution (plasma/ serum) as a novel IFN-inducible protein biomarker in SLE. Two factors have kindled our interest in G3BP. First, the gene encoding for G3BP is part of the original IFN gene signature in SLE PBMCs. ${ }^{4}$ Second, in patients with SLE we have recently discovered a specific upregulation of G3BP along with ICs in circulating cell-derived MPs, putative major sources of extracellular autoantigens in SLE. ${ }^{12-15}$ The MP-G3BP upregulation was most pronounced in patients with active lupus nephritis. The formation and functions of G3BP are not well-defined. G3BP is an extracellular protein, is widely expressed in most tissues and has been detected in serum, breast milk and semen. ${ }^{16}{ }^{17}$ In vitro double-stranded polynucleotides, $\gamma$-IFN, thyroid stimulating hormone (TSH), lipopolysaccharide (LPS), and insulin/insulin-like growth factor (IGF)-I trigger G3BP production. ${ }^{18}{ }^{19}$ Circulating G3BP is likely to reflect type I IFN activity but this has not yet to our knowledge been investigated in cohorts of patients with SLE. Other factors may regulate the formation and fate of G3BP and, as expected, plasma G3BP has been found elevated during chronic viral infections, and in various types of solid cancers, Behcet's disease, rheumatoid arthritis and SLE. ${ }^{20-25}$ In the latter study, the patients with SLE were included as disease controls, and clinical associations were not explored. $^{22}$

In the present study, we quantify G3BP in solution (plasma/serum) and explore the potential of G3BP to serve as a biomarker of type I IFN activity, disease activity, and clinical and serological manifestations investigating independent cross-sectional and longitudinal SLE cohorts.

\section{MATERIAL AND METHODS}

\section{Patients and controls}

Four separate and independent SLE-cohorts were included in the study. Three cohorts were cross-sectional and were named: SLE-IFN- $\alpha$, SLE-Denmark (DK), and SLE-Sweden (SE). These cohorts also included healthy controls (HCs) as described below. In the fourth longitudinal cohort named SLE-L, consecutive samples with disease activity scores were available. No matching controls were available for the consecutive samples. All patients in the SLE-IFN- $\alpha$, SLE-DK and SLE-SE fulfilled at least four of the 1997 revised American College of Rheumatology (ACR) criteria for SLE. ${ }^{26}$ Patient samples were collected from 1995 through to 2000 in the SLE-L cohort and classified according to the revised 1982 ACR classification criteria for SLE. ${ }^{27}$ In the SLE-DK cohort $(\mathrm{n}=70) 6$ male and 64 female patients with SLE were included and had a median age of 39 years (range 21-76 years) and a median disease duration of 10 years (range 0-37 years). The SLE-SE cohort serves as an ethnically comparable validation cohort for plasma G3BP concentrations and clinical correlates. The SLE-SE cohort included 9 male and 59 female patients $(\mathrm{n}$, total $=68$ ) with a median age of 53 years (range 20-84 years) and a median disease duration of 13 years (range $0-49$ years). The SLE-IFN- $\alpha$ cohort included 26 female patients with a median age of 41 years (range 19-70 years) and high disease activity to explore the correlations between G3BP and type I IFN activity. In the SLE-L cohort, serum and disease activity scores were available from three or four consecutive visits at least 6 months apart in 13 female and 2 male patients with a median age at inclusion of 29 years (range 16-57 years). These visits were conducted from 1995 through to 2000. All patients with SLE were Caucasian except one in the SLE-DK cohort.

Patients that fulfilled the ACR criteria for systemic sclerosis (SSc, n=111) were included as disease controls for the plasma G3BP concentrations. ${ }^{28}$ Sixteen men and 95 women were included and the median age was 60 years (range 22-79 years). Seventy-four patients had limited cutaneous SSc and 37 had diffuse cutaneous SSc.

Three independent cohorts of non-medicated, agematched and gender-matched HCs were included and named according to the SLE-cohort nomenclature above, that is, HC-IFN- $\alpha$, HC-DK and HC-SE. The HC-IFN- $\alpha$ includes 10 female HCs with a median age of 28 years (range $24-48$ years). The HC-DK cohort $(n=47)$ consists of 41 women and 6 men with a median age of 45 years (range 24-62 years) and the HC-SE cohort consists of only women $(\mathrm{n}=50)$ with a median age of 50 years (range $20-63$ years).

The research protocol was approved by the local ethics committees and carried out in accordance with the principles of the Declaration of Helsinki. All human participants provided written informed consent.

\section{Clinical and paraclinical assessment}

Clinical characteristics of the patients with SLE in the SLE-DK, SLE-SE and SLE-IFN- $\alpha$ cohorts are summarised in table 1 . Disease activity was scored by the SLE Disease Activity Index 2000 (SLEDAI 2K), except for the SLE-L cohort which was scored by the original SLEDAI. ${ }^{29}{ }^{30}$ 
Table 1 Clinical characteristics of patients with systemic lupus erythematosus (SLE) in the SLE-DK (plasma), SLE-SE (plasma), and SLE-IFN- $\alpha$ (serum) cohorts ${ }^{\star}$

\begin{tabular}{|c|c|c|c|}
\hline & SLE-DK (n=70) & SLE-SE $(n=68)$ & SLE-IFN- $\alpha(n=26)$ \\
\hline \multicolumn{4}{|l|}{ Disease manifestations $†$} \\
\hline Renal disease & $12(17)$ & $12(18)$ & $13(50)$ \\
\hline Arthritis & $7(10)$ & $7(10)$ & $8(31)$ \\
\hline Rash & $4(6)$ & $5(7)$ & $10(14)$ \\
\hline Vasculitis & $4(6)$ & $2(3)$ & $1(4)$ \\
\hline Alopecia & $5(7)$ & $4(6)$ & $3(12)$ \\
\hline Mucosal ulcers & $6(9)$ & $3(4)$ & $7(27)$ \\
\hline Serositis & $2(3)$ & $0(0)$ & $5(19)$ \\
\hline Leucopenia & $8(11)$ & $3(4)$ & $4(15)$ \\
\hline Thrombocytopenia & $3(4)$ & $1(1)$ & $6(23)$ \\
\hline SLEDAI, mean $\pm S D$ (range) $†$ & $5 \pm 5(0-21)$ & $4 \pm 4(0-14)$ & $9 \pm 7(0-20)$ \\
\hline \multicolumn{4}{|l|}{ Autoantibodies and complement $†$} \\
\hline Anti-dsDNA & $30(43)$ & $15(22)$ & $10(38)$ \\
\hline Anti-histone & $48(69)$ & ND & ND \\
\hline Anti-ENA & $14(20)$ & ND & ND \\
\hline Anti-C1q & $17(24)$ & ND & ND \\
\hline Low C3 or C4 & $49(70)$ & $20(29)$ & $9(35)$ \\
\hline \multicolumn{4}{|l|}{ Medication† } \\
\hline Prednisolone $\leq 7.5 \mathrm{mg}$ daily & $16(23)$ & $35(51)$ & $10(38)$ \\
\hline Prednisolone $>7.5 \mathrm{mg}$ daily & $14(20)$ & $14(21)$ & $8(31)$ \\
\hline Antimalarials & $13(19)$ & $45(66)$ & $5(19)$ \\
\hline Azathioprine, MTX or mycophenylate mofetil & $30(43)$ & $30(44)$ & $15(58)$ \\
\hline SLICC/ACR Damage Index, mean \pm SD (range)† & $1 \pm 1(0-8)$ & $2 \pm 2(0-11)$ & ND \\
\hline Antiphospholipid syndrome & $16(23)$ & $20(29)$ & ND \\
\hline \multicolumn{4}{|c|}{$\begin{array}{l}\text { *Values are numbers (per cent) of subjects unless otherwise stated. } \\
\text { †At study inclusion. } \\
\text { †Values below the reference interval of the assay. Disease manifestations are recorded items in the SLEDAI and thus represent } \\
\text { manifestations of active disease. } \\
\text { C1q, complement component C1q; C3, complement component C3; C4, complement component C4; DK, Denmark; dsDNA, double-stranded } \\
\text { DNA; ENA, extractable nuclear antigen; IFN- } \alpha \text {, interferon- } \alpha \text {; MTX, methotrexate; ND, not determined; SE, Sweden; SLEDAI, Systemic Lupus } \\
\text { Erythematosus Disease Activity Index; SLICC/ACR, Systemic Lupus International Collaborating Clinics/American College of Rheumatology. }\end{array}$} \\
\hline
\end{tabular}

Cumulative organ damage was assessed using the Systemic Lupus International Collaborating Clinics Damage Index score. ${ }^{31}$ Baseline clinical manifestations at time of inclusion and blood collection were recorded according to the definitions of SLEDAI. Patients were categorised as either having or not having active nephritis at inclusion for the correlation analysis based on proteinuria lower/higher than $0.5 \mathrm{~g} / 24 \mathrm{~h}$.

Routine clinical biochemistry parameters were obtained for all patient samples. Antinuclear antibodies were detected by indirect immunofluorescence on HEp-2 cells in the patients with SLE and SSc. Antibodies against double-stranded DNA were determined by ELISA. The SLE-DK samples were also screened for antiextractable nuclear antigen using the Diastat ENA singlewell screen ELISA from Euro-Diagnostica (Malmö, Sweden). In SLE-DK anti-phospholipid antibodies (anti-cardiolipin IgG/IgM and $\beta_{2}$ glycoprotein-I IgG/IgM) and antibodies against histones, Clq, Ro60, Ro52, and La were measured by ELISA. Anti-Scl-70 antibodies were detected with line-immunoassay in the patients with SSc. Complement C3 and C4 were measured with immunonephelometry on an Immage System (Beckman-Coulter, Fullerton, California, USA).

\section{Biological samples}

EDTA plasma (SLE-DK and SLE-SE) and serum (SLE-INF- $\alpha$ and SLE-L) were separated according to routine protocols and stored at $-80^{\circ} \mathrm{C}^{32}$

\section{Quantification of G3BP using ELISA}

EDTA plasma or serum samples were thawed, diluted 1:100 in Sample Diluent and analysed in duplicate according to the manufacturer's instructions using the Human 90K/Mac-2BP Platinum ELISA kit (BMS234, Bender MedSystems, Vienna, Austria). Plasma and serum concentrations were expressed in $\mu \mathrm{g} / \mathrm{mL}$ plasma. The intra-assay coefficient of variation was estimated to $5.7 \%$. The interassay coefficients of variation were determined in one HC and one SLE sample analysed in duplicate on five plates and were $6.5 \%$ and $9.7 \%$, respectively.

\section{Type I IFN activity in serum and PBMCs}

In the SLE-IFN- $\alpha$ and HC-IFN- $\alpha$ cohort IFN activity was indirectly measured by a $\mathrm{Mx} 1$ driven luciferase reporter gene assay and by IFN- $\alpha$ inducible gene expression scores from PBMCs as presented previously. ${ }^{33}$ The Mx1 driven luciferase reporter gene assay was based on the 93D 7 cell line derived by stable transfection of the A549 cell line 
(CLL-185, ATCC) with an IFN-inducible construct, harbouring the $\mathrm{Mx} 1$ promotor driving a luciferase reporter gene, which was provided by ARGOS (who obtained it from Dr Guenther Adolf, Boehringer-Ingelheim Gmbh, Austria). Previously, similar Mx1-driven reporter gene assays were shown to be specific, precise and linear in the range $1-16$ IFN IU $/ \mathrm{mL} .{ }^{34}$ To increase the specificity and sensitivity for IFN- $\alpha$ activity, a combined expression score of 12 IFN- $\alpha$ inducible genes in PBMCs was determined using microarray technology (GeneChip Human Genome U133 Plus 2.0 Array, Affymetrix, Santa Clara, California). Briefly, for each of these 12 previously defined IFN-inducible genes (IFI44L, OAS1, RSAD2, MX1, IFI27, PLSCR1, IFIT1, IFIT3, OAS2, IFIT2, USP18 and PSTI1, not including the gene for G3BP), a Z-score (standard score) was calculated across the controls or the patients; and then the Z-scores for the 12 genes were averaged to a single score.

\section{Statistical analysis}

G3BP concentrations were non-normally distributed and are presented throughout the manuscript with median and 5-95th centiles (median, 5-95th centiles). In the consecutive cohort, changes $(\Delta)$ in G3BP and SLEDAI values between visits were calculated and subjected to correlation analysis. For statistical comparisons, Mann-Whitney's U test was used to compare two groups separately to evaluate $p$ values. Univariate correlation analyses were performed by calculating Spearman's rank correlation coefficients (r). No adjustments for multiple comparisons were made. $p$ Values less than 0.05 were considered statistically significant. GraphPad Prism V.5 (GraphPad Software, San Diego, California, USA) was used for the statistical calculations and plots.

\section{RESULTS}

Correlations between serum G3BP and type I IFN activity

The serum G3BP concentrations correlated significantly with the activity scores from the Mx1-reporter assay and the calculated IFN- $\alpha$ scores (IFN-induced gene expression in PBMCs) $(\mathrm{r}=0.56, \mathrm{p}=0.0005$ and $\mathrm{r}=0.58, \mathrm{p}=0.0002$, respectively) corroborating the notion that G3BP reflects type I IFN activity (figure 1A,B). Also, the serum concentrations of G3BP were significantly increased in the patients with SLE compared with HCs $(7.7 \mu \mathrm{g} / \mathrm{mL}, 3.6-$ 17.3 vs $5.2 \mu \mathrm{g} / \mathrm{mL}, 2.4-7.8 ; \mathrm{p}=0.007$, data not shown).

\section{Plasma G3BP in patients with SLE compared with patients with SSC and HCS}

As shown in figure 2, the G3BP plasma concentrations were significantly increased in the two independent SLE cohorts (SLE-DK: $5.4 \mu \mathrm{g} / \mathrm{mL}, 2.3-15.5$, and SLE-SE: $4.5 \mu \mathrm{g} / \mathrm{mL}, 2.1-12.0)$ compared with patients with SSc $(3.7 \mu \mathrm{g} / \mathrm{mL}, 1.8-8.0) \quad(\mathrm{p}<0.001$ in both cases $)$, and with the two HC groups (HC-DK: $2.7 \mu \mathrm{g} / \mathrm{mL}, 1.1-6.3$, and HC-SE: $3.1 \mu \mathrm{g} / \mathrm{mL}, 1.9-8.1) \quad(\mathrm{p}<0.0001$ also in both cases). Patients with SSc were intermediate between the HCs and patients with SLE and have significantly increased concentrations compared with both $\mathrm{HC}$ cohorts $(\mathrm{p}<0.05)$. Patients with limited $(\mathrm{n}=74)$ and diffuse $(n=37)$ cutaneous SSc did not differ in their plasma levels ( $\mathrm{p}=0.96$, data not shown). Comparing median G3BP levels in serum and plasma samples we note generally higher levels in sera while differences between SLE and HCs persist in both types of biological samples.

\section{Longitudinal changes in G3BP serum concentrations}

To explore the longitudinal change in IFN activity and association with disease activity, we included 15 patients with three or four longitudinal samples (at least 6 months apart) available and with high disease activity at minimum one visit. G3BP levels and disease activity (SLEDAI) changed markedly over time and appeared to correlate in most of the 15 patients (figure 3) supported by a significant association between changes in G3BP and SLEDAI between each visit (Spearman's $r=0.44$, $\mathrm{p}=0.014$, data not shown).
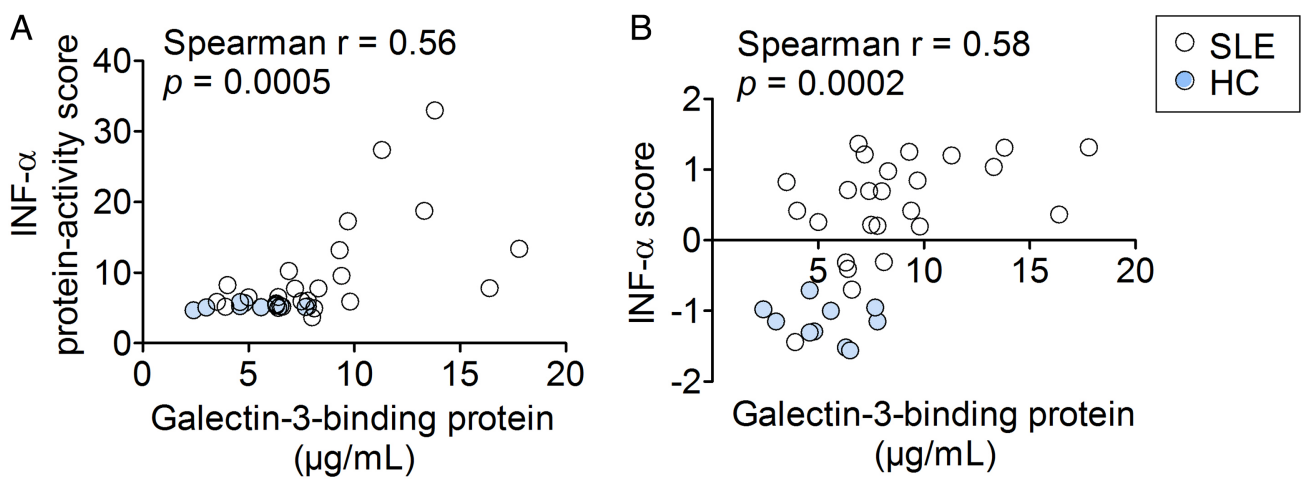

Figure 1 Correlations between two measures of type I interferon activity and serum galectin-3-binding protein in the SLE-IFN- $\alpha$ $(n=26)$ and HC-IFN- $\alpha(n=10)$ cohorts. (A) The IFN- $\alpha$ protein-activity scores obtained using the Mx-1 gene reporter assay and (B) the calculated IFN- $\alpha$ scores representing gene expression of IFN-inducible genes in PBMCs correlated significantly with serum concentrations of galectin-3-binding protein. Non-parametric correlation analysis (Spearman's $r$ ) was used to estimate correlations. IFN- $\alpha$, interferon- $\alpha$; HC, healthy controls; SLE, systemic lupus erythematosus; PBMCs, peripheral blood mononuclear cells. 


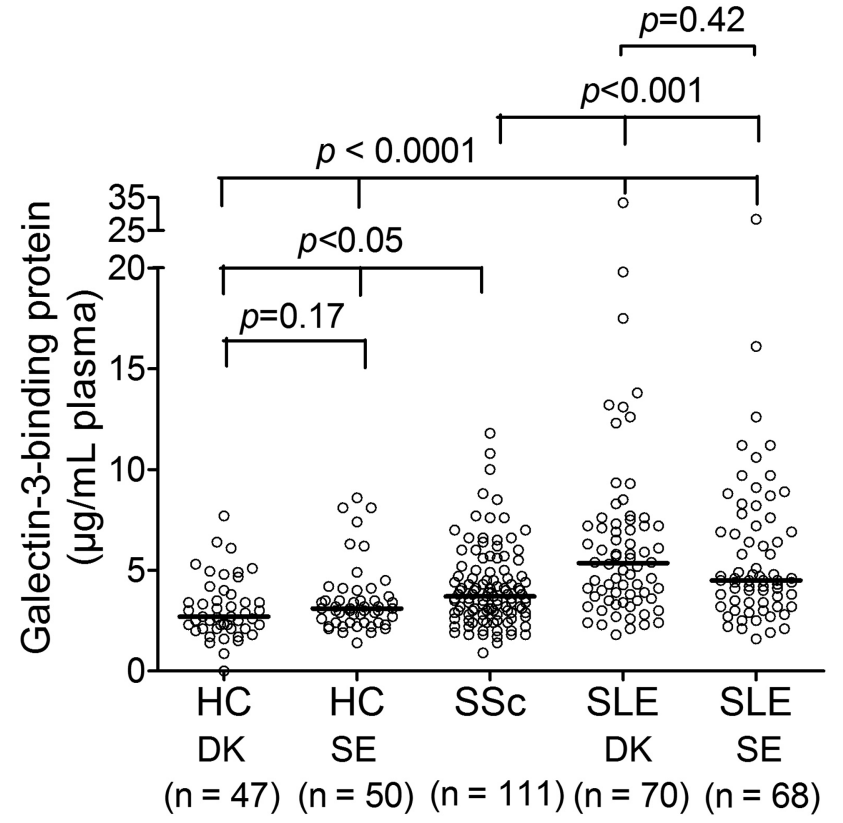

Figure 2 Plasma galectin-3-binding protein concentrations in $\mathrm{HCs}$ and patients with SLE and SSc. Highly significantly increased plasma concentrations of G3BP were found in both SLE-cohorts compared with the patients with SSc and both $\mathrm{HC}$ cohorts. This observation was confirmed in the independent validation SLE-SE and HC-SE cohorts. Bars depict the median. Comparisons were performed with the Mann-Whitney $U$ test to compare two groups in order to obtain the specific $p$ values. The presented $p$ values are not adjusted to multiple comparisons. G3BP, galectin-3-binding protein; HC, healthy control; SSc, systemic sclerosis; SLE, systemic lupus erythematosus; DK, Denmark; SE, Sweden.

\section{Correlations between G3BP levels and disease activity, and clinical and serological parameters}

In the SLE-DK cohort, a significant association was observed between plasma G3BP and the presence of autoantibodies against double-stranded DNA, Ro, La and Sm-antigen, high disease activity (SLEDAI), erythrocyte sedimentation rate, and haematological abnormalities (low haemoglobin and neutrophil concentrations), but not with having active nephritis at inclusion (table 2). In the smaller and highly active SLE-IFN- $\alpha$ cohort, we observed a significant correlation with anti-dsDNA levels, SLEDAI and active nephritis, but not with haematological abnormalities and autoantibodies against ribonucleoproteins had not been tested. None of these associations could be confirmed in the SLE-SE cohort.

\section{DISCUSSION}

In the present study we hypothesised that G3BP, an IFN-inducible protein in PBMCs with increased expression in circulating MPs in SLE plasma, may serve as a novel IFN-induced protein biomarker in SLE. We demonstrate that the level of circulating G3BP reflects type I IFNs and, as expected, plasma concentrations are significantly increased in two large, independent SLE cohorts compared with disease cohorts and HCs. In two SLE cohorts the levels were associated with the presence of antinuclear antibodies, specific disease manifestations and high disease activity in line with previous reports of type I IFNs in SLE. In a cross-sectional cohort we observed that G3BP fluctuated markedly over time and also correlated with disease activity measures. However, these associations could not be confirmed in the cross-sectional validation cohort. Thus G3BP provides a new and very convenient tool to assess type I IFN activity, while its role as a single diagnostic or disease activity biomarker needs further investigation.

We demonstrated that G3BP levels correlated well with two validated indirect measures of type I IFN activity. ${ }^{4} 34$ Accordingly, G3BP reflects type I IFN activity and seems able to identify IFN-high individuals with the same accuracy as the gene reporter assay. In the case of gene expression scores from PBMCs we find an almost complete discrimination of all patients with SLE from HCs. This suggests that gene expression scores are more sensitive and more specific markers of type I IFN activation, but the analysis is also more labour intensive, expensive and requires PBMCs in contrast to the G3BP analysis. Sampling is uncomplicated and like other IFN-induced proteins G3BP may be less affected by fluctuations in leucocyte levels, and could prove a sensitive measure of disease activity and predictors of flares like IP-10 or SIGLEC-1. ${ }^{7} 10$ However, the strong association with disease activity measures of G3BP as well as the other cytokines/chemokines may reflect regulation from other inflammatory cytokines such as tumour necrosis factor and IFN- $\gamma$. G3BP may provide additional information to the chemokines and SIGLEC- 1 , and combinations of these measures could improve their sensitivity and specificity in the future.

Using two large independent SLE cohorts we demonstrated significantly elevated levels of G3BP in plasma compared with HCs and a large cohort of patients with SSc. G3BP has been detected in most body fluids and is expressed in most tissues. ${ }^{16}{ }^{17}$ Higher levels of G3BP in serum from patients with SLE and HCs compared with plasma measurements suggested that plasma and serum are not directly comparable. Increased levels of G3BP have previously been demonstrated in SLE and rheumatoid arthritis (RA) included as control groups in a study of patients with Bechet's disease. ${ }^{22}$ Others did not find increased serum concentrations of G3BP in patients with RA compared with HCs and patients with osteoarthritis, however, high levels were found in the synovial fluid as well as a high expression in the synovial lining. ${ }^{23}$ Increased activation of the type I IFN system is a common denominator in rheumatic autoimmune diseases and is most pronounced in SLE and polymyositis/ dermatomyositis and less in SSc and RA. ${ }^{35}$ This is in line with our findings of G3BP levels in patients with SSc being intermediate to SLE and HCs. 


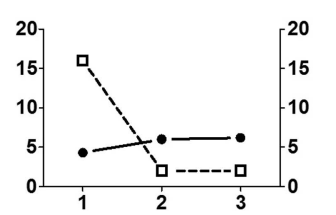

- G3BP $(\mu \mathrm{g} / \mathrm{mL})$

Ð SLEDAI
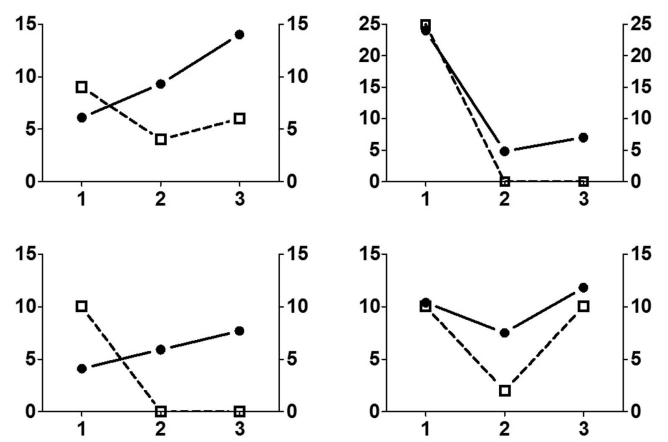

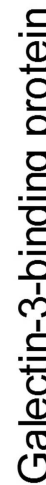
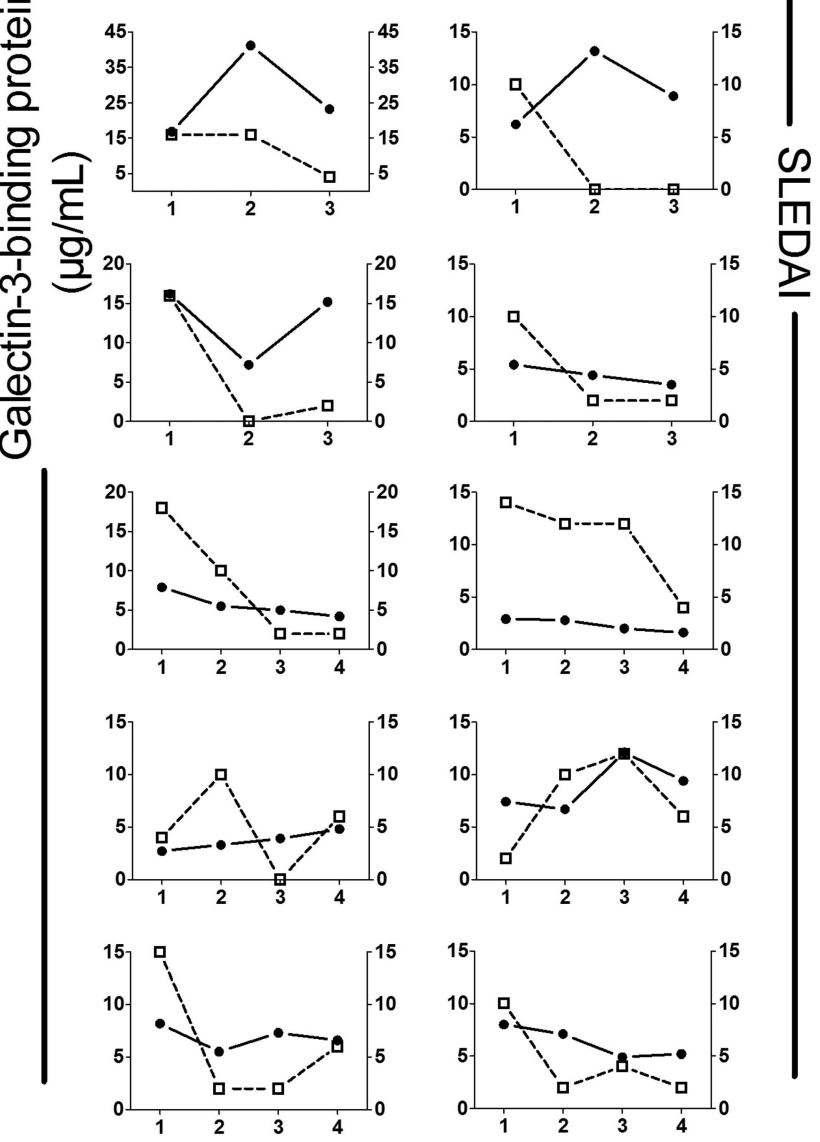

Visit

Figure 3 Longitudinal changes in serum G3BP concentrations and SLEDAI in 15 patients with SLE. Each plot represents one patient. Temporal change in G3BP was observed for all 15 patients weakly associated with SLEDAI. G3BP, galectin-3-binding protein; SLE, systemic lupus erythematosus; SLEDAI, systemic lupus erythematosus disease activity index.

The significant associations in the DK cohort of G3BP with autoantibody profiles, high disease activity (SLEDAI), low haemoglobin and neutrophils, and high erythrocyte sedimentation rate are in line with the majority of the studies regarding type I IFN activity. ${ }^{4-7} 36-$ 38 In particular, type I IFNs are strongly linked to subgroups of patients with specific serological profiles and specific autoantibodies against ribonucleoproteins, rather than to specific disease manifestations or high disease activity. ${ }^{5}$ This may also hold true for G3BP. In the SLE-IFN- $\alpha$ cohort, the presence of anti-dsDNA antibodies was highly significantly associated with the G3BP levels, while, unfortunately, anti-Ro, anti-La or anti-Sm had not been determined in this cohort. Only a weak association with SLEDAI was found in the SLE-IFN- $\alpha$ cohort which is characterised by higher disease activity than the other cohorts. Over time in the consecutive samples G3BP and SLEDAI also seemed to correlate. It was surprising that we could not confirm the SLEDAI association in the SLE-SE cohort, since the number of patients, their characteristics and their G3BP levels overall were very similar to the DK-cohort. However, the DK-cohort had higher SLEDAI scores, an increased proportion were anti-dsDNA positive and had low complement levels, and fewer received antimalarials. The discrepancies in the clinical correlates may thus be explained by a low number of active patients in the SE-cohort, but may also reflect the effects of antimalarials on IC-driven disease activity and IFN response during flares. Besides disease activity, type I IFN activation, medical treatment and timing of sampling during flares, and other so far unknown factors that control the formation and fate of G3BP, are likely to affect its levels. The previous G3BP study that included patients with SLE as controls did not explore clinical or serological correlates. ${ }^{22}$ Thus additional studies of G3BP as a biomarker of disease activity and specific disease manifestations are needed to resolve these issues.

Fluctuation of type I IFN activation over time and the factors that influence this including the association with disease activity is still elusive. ${ }^{6}{ }^{8}{ }^{39}$ Bengtsson et al observed that type I IFN activation changed with disease activity, while this was not the case in the studies by Landolt-Marticorena et $a \rho^{39}$ and Petri et $a l^{8}$ Landolt-Marticorena et al observed fluctuations in type I IFN activation at sample intervals from 3 months up to more than a year, and Petri et al found that type I IFN activation were stable from patient to patient at shorter sampling intervals (less than 3 months). This could reflect that type I IFNs are more linked to the autoantibody profiles that tend to remain more stable over time than to overall disease activity. ${ }^{5}$ In our longitudinal study we observed distinct changes in G3BP that were associated with global disease activity measures. Unfortunately, we did not have access to other factors such as medication as well as specific components of the SLEDAI scores or serological profiles to explore the association between these and G3BP over time in this cohort of patients.

In conclusion, G3BP levels in the circulation are increased in patients with SLE possibly as a reflection 
Table 2 Correlation analysis of plasma galectin-3-binding protein (plasma/serum) and clinical/serological parameters with Spearman's rank correlation coefficients $(r)$ and $p$ values

\begin{tabular}{|c|c|c|c|c|c|c|c|c|c|}
\hline & \multicolumn{3}{|c|}{ SLE-DK (plasma) } & \multicolumn{3}{|c|}{ SLE-SE (plasma) } & \multicolumn{3}{|c|}{ SLE-INF- $\alpha$ (serum) } \\
\hline & $\overline{\mathbf{n}}$ & $\mathbf{r}$ & p Value & $\overline{\mathbf{n}}$ & $\mathbf{r}$ & p Value & $\overline{\mathbf{n}}$ & $\mathbf{r}$ & p Value \\
\hline Age & 70 & -0.32 & 0.01 & 68 & 0.0018 & 0.99 & 26 & -0.16 & 0.43 \\
\hline Nephritis* & 70 & 0.14 & 0.24 & 68 & 0.049 & 0.69 & 26 & 0.42 & 0.03 \\
\hline SLEDAI* & 70 & 0.41 & 0.0004 & 68 & -0.0025 & 0.98 & 26 & 0.38 & 0.05 \\
\hline SLICC/ACR Damage Index & 70 & -0.08 & 0.53 & 68 & 0.036 & 0.77 & & $\mathrm{~N} / \mathrm{A}$ & \\
\hline Immunosuppressive treatment $†$ & 70 & -0.05 & 0.68 & 68 & 0.052 & 0.68 & 26 & 0.29 & 0.16 \\
\hline ESR & 66 & 0.57 & $<0.0001$ & & $\mathrm{~N} / \mathrm{A}$ & & & N/A & \\
\hline Haemoglobin & 70 & -0.29 & 0.02 & 68 & -0.21 & 0.08 & 26 & 0.17 & 0.41 \\
\hline Neutrophils & 70 & -0.36 & 0.002 & 67 & 0.037 & 0.77 & & N/A & \\
\hline Lymphocytes & 70 & -0.04 & 0.72 & 67 & -0.084 & 0.50 & 26 & 0.11 & 0.58 \\
\hline Platelets & 70 & -0.02 & 0.85 & & $\mathrm{~N} / \mathrm{A}$ & & 26 & -0.06 & 0.78 \\
\hline Anti-dsDNA & 69 & 0.35 & 0.003 & 68 & -0.094 & 0.45 & 26 & 0.54 & 0.005 \\
\hline Anti-Sm & 69 & 0.26 & 0.03 & 68 & 0.0021 & 0.99 & & $\mathrm{~N} / \mathrm{A}$ & \\
\hline Anti-Ro & 69 & 0.32 & 0.01 & & $\mathrm{~N} / \mathrm{A}$ & & & $N / A$ & \\
\hline Anti-La & 69 & 0.25 & 0.04 & & N/A & & & N/A & \\
\hline C3 low & 69 & -0.03 & 0.78 & 68 & 0.095 & 0.44 & 26 & -0.30 & 0.14 \\
\hline C4 low & 69 & -0.09 & 0.45 & 68 & 0.14 & 0.26 & 26 & -0.28 & 0.17 \\
\hline
\end{tabular}

Bold denotes significant observations $(p<0.05)$.

${ }^{*}$ All of the clinical manifestations included in the SLEDAI were tested and no other significant correlations were detected.

†At inclusion. Immunosuppressive treatment included prednisolone, methotrexate, mycophenylate mofetil, azathioprine and plaquenil.

C3, complement component C3; C4, complement component C4; DK, Denmark; ESR, erythrocyte sedimentation rate; N/A, not available; IFN- $\alpha$, interferon- $\alpha$; SE, Sweden; SLEDAI, Systemic Lupus Erythematosus Disease Activity Index; SLICC/ACR, Systemic Lupus International Collaborating Clinics/American College of Rheumatology.

of increased type I IFN activity. G3BP provides a convenient indirect measure of type I IFNs. Although promising, the clinical and disease activity correlates with G3BP were discrepant, and further studies are needed to clarify the role of G3BP as a biomarker in SLE.

\section{Author affiliations}

${ }^{1}$ Department of Clinical Biochemistry, Immunology \& Genetics, Statens Serum Institut, Copenhagen, Denmark

${ }^{2}$ Department of Infectious Diseases \& Rheumatology, Rigshospitalet,

Copenhagen University Hospital, Copenhagen, Denmark

${ }^{3}$ Department of Rheumatology, Skåne University Hospital and Lund University, Lund, Sweden

${ }^{4}$ Department of Dermatology, Bispebjerg, Copenhagen University Hospital, Copenhagen, Denmark

${ }^{5}$ Department of Rheumatology, Odense University Hospital, Odense, Denmark

Funding This research was supported by The Danish Rheumatism Association Grant R99-A1937 and The Foundation for the Advancement of Medical Science (Heegaard), The Danish Rheumatism Association (Grant R33-A1836), the AP Møller Foundation, and The Region of Southern Denmark (Voss), the Novo Nordisk Research Foundation (Jacobsen). Medical Faculty at Lund University, Alfred Österlund's Foundation, the Crafoord Foundation, the Swedish Rheumatism Association, Greta and Johan Kock's Foundation, King Gustaf V's 80th Birthday Foundation, the Swedish Society of Medicine, the Foundation of the National Board of Health and Welfare and Skåne University Hospital, Lund, Sweden (Lood, Bengtsson)

Competing interests None.

Ethics approval Ethics Committee, Capital Region Copenhagen.

Provenance and peer review Not commissioned; externally peer reviewed.

Data sharing statement No additional data are available.

Open Access This is an Open Access article distributed in accordance with the Creative Commons Attribution Non Commercial (CC BY-NC 3.0) license, which permits others to distribute, remix, adapt, build upon this work noncommercially, and license their derivative works on different terms, provided the original work is properly cited and the use is non-commercial. See: http:// creativecommons.org/licenses/by-nc/3.0/

\section{REFERENCES}

1. Obermoser G, Pascual V. The interferon-alpha signature of systemic lupus erythematosus. Lupus 2010;19:1012-9.

2. Rahman A, Isenberg DA. Systemic lupus erythematosus. $N$ Engl $J$ Med 2008;358:929-39.

3. Hooks JJ, Moutsopoulos HM, Geis SA, et al. Immune interferon in the circulation of patients with autoimmune disease. $N$ Engl J Med 1979;301:5-8.

4. Baechler EC, Batliwalla FM, Karypis G, et al. Interferon-inducible gene expression signature in peripheral blood cells of patients with severe lupus. Proc Natl Acad Sci USA 2003;100:2610-5.

5. Weckerle CE, Franek BS, Kelly JA, et al. Network analysis of associations between serum interferon-alpha activity, autoantibodies, and clinical features in systemic lupus erythematosus. Arthritis Rheum 2011;63:1044-53.

6. Bengtsson AA, Sturfelt G, Truedsson L, et al. Activation of type I interferon system in systemic lupus erythematosus correlates with disease activity but not with antiretroviral antibodies. Lupus 2000;9:664-71.

7. Bauer JW, Petri M, Batliwalla FM, et al. Interferon-regulated chemokines as biomarkers of systemic lupus erythematosus disease activity: a validation study. Arthritis Rheum 2009;60:3098-107.

8. Petri M, Singh S, Tesfasyone $\mathrm{H}$, et al. Longitudinal expression of type I interferon responsive genes in systemic lupus erythematosus. Lupus 2009;18:980-9.

9. Bennett L, Palucka AK, Arce E, et al. Interferon and granulopoiesis signatures in systemic lupus erythematosus blood. J Exp Med 2003;197:711-23.

10. Rose T, Grutzkau A, Hirseland $\mathrm{H}$, et al. IFNalpha and its response proteins, IP-10 and SIGLEC-1, are biomarkers of disease activity in systemic lupus erythematosus. Ann Rheum Dis 2013;72:1639-45.

11. Merrill JT, Wallace DJ, Petri M, et al. Safety profile and clinical activity of sifalimumab, a fully human anti-interferon alpha monoclonal antibody, in systemic lupus erythematosus: a phase I, multicentre, double-blind randomised study. Ann Rheum Dis 2011;70:1905-13. 
12. Ostergaard $\mathrm{O}$, Nielsen $\mathrm{CT}$, Iversen LV, et al. Unique protein signature of circulating microparticles in systemic lupus erythematosus. Arthritis Rheum 2013;65:2680-90.

13. Ullal AJ, Reich CF III, Clowse M, et al. Microparticles as antigenic targets of antibodies to DNA and nucleosomes in systemic lupus erythematosus. J Autoimmun 2011;36:173-80.

14. Beyer C, Pisetsky DS. The role of microparticles in the pathogenesis of rheumatic diseases. Nat Rev Rheumatol 2010;6:21-9.

15. Nielsen CT, Ostergaard O, Stener L, et al. Increased IgG on cell-derived plasma microparticles in systemic lupus erythematosus is associated with autoantibodies and complement activation. Arthritis Rheum 2012;64:1227-36.

16. Sasaki T, Brakebusch C, Engel J, et al. Mac-2 binding protein is a cell-adhesive protein of the extracellular matrix which self-assembles into ring-like structures and binds beta1 integrins, collagens and fibronectin. EMBO J 1998;17:1606-13.

17. Ullrich $A$, Sures I, D'Egidio M, et al. The secreted tumor-associated antigen $90 \mathrm{~K}$ is a potent immune stimulator. $\mathrm{J}$ Biol Chem 1994;269:18401-7.

18. Grassadonia A, Tinari N, Fiorentino B, et al. The $90 \mathrm{~K}$ protein increases major histocompatibility complex class I expression and is regulated by hormones, gamma-interferon, and double-strand polynucleotides. Endocrinology 2004;145:4728-36.

19. Shaked I, Hanna DB, Gleissner C, et al. Macrophage inflammatory markers are associated with subclinical carotid artery disease in women with human immunodeficiency virus or hepatitis $\mathrm{C}$ virus infection. Arterioscler Thromb Vasc Biol 2014;34:1085-92.

20. Cheung KJ, Libbrecht L, Tilleman K, et al. Galectin-3-binding protein: a serological and histological assessment in accordance with hepatitis C-related liver fibrosis. Eur J Gastroenterol Hepatol 2010;22:1066-73.

21. lacobelli S, Sismondi $\mathrm{P}$, Giai M, et al. Prognostic value of a novel circulating serum $90 \mathrm{~K}$ antigen in breast cancer. $\mathrm{Br} J$ Cancer 1994;69:172-6.

22. Lee YJ, Kang SW, Song JK, et al. Serum galectin-3 and galectin-3 binding protein levels in Behcet's disease and their association with disease activity. Clin Exp Rheumatol 2007;25(4 Suppl 45):S41-5.

23. Ohshima S, Kuchen S, Seemayer CA, et al. Galectin 3 and its binding protein in rheumatoid arthritis. Arthritis Rheum 2003:48:2788-95.

24. Tinari N, Natoli C, D'Ostilio N, et al. 90K (Mac-2 BP) predicts CD4 decline in human immunodeficiency virus-infected patients with CD4 counts above 200×10(6) cells/L. Arch Pathol Lab Med 1998;122:178-81.

25. Zeimet AG, Natoli C, Herold M, et al. Circulating immunostimulatory protein $90 \mathrm{~K}$ and soluble interleukin-2-receptor in human ovarian cancer. Int J Cancer 1996;68:34-8.
26. Hochberg MC. Updating the American College of Rheumatology revised criteria for the classification of systemic lupus erythematosus. Arthritis Rheum 1997;40:1725.

27. Tan EM, Cohen AS, Fries JF, et al. The 1982 revised criteria for the classification of systemic lupus erythematosus. Arthritis Rheum 1982;25:1271-7.

28. Subcommittee for scleroderma criteria of the American Rheumatism Association Diagnostic and Therapeutic Criteria Committee. Preliminary criteria for the classification of systemic sclerosis (scleroderma). Arthritis Rheum 1980;23:581-90.

29. Bombardier C, Gladman DD, Urowitz MB, et al. Derivation of the SLEDAI. A disease activity index for lupus patients. The Committee on Prognosis Studies in SLE. Arthritis Rheum 1992;35:630-40.

30. Gladman DD, Ibanez D, Urowitz MB. Systemic lupus erythematosus disease activity index 2000. J Rheumatol 2002;29:288-91.

31. Gladman D, Ginzler E, Goldsmith C, et al. The development and initial validation of the Systemic Lupus International Collaborating Clinics/American College of Rheumatology damage index for systemic lupus erythematosus. Arthritis Rheum 1996;39:363-9.

32. Nielsen CT, Ostergaard O, Johnsen C, et al. Distinct features of circulating microparticles and their relationship to clinical manifestations in systemic lupus erythematosus. Arthritis Rheum 2011;63:3067-77.

33. Hermansen ML, Hummelshoj L, Lundsgaard D, et al. Increased serum beta2-microglobulin is associated with clinical and immunological markers of disease activity in systemic lupus erythematosus patients. Lupus 2012;21:1098-104.

34. Canosi U, Mascia M, Gazza L, et al. A highly precise reporter gene bioassay for type I interferon. J Immunol Methods 1996;199:69-76.

35. Higgs BW, Liu Z, White B, et al. Patients with systemic lupus erythematosus, myositis, rheumatoid arthritis and scleroderma share activation of a common type I interferon pathway. Ann Rheum Dis 2011;70:2029-36.

36. Kirou KA, Lee C, George S, et al. Activation of the interferon-alpha pathway identifies a subgroup of systemic lupus erythematosus patients with distinct serologic features and active disease. Arthritis Rheum 2005;52:1491-503.

37. Nikpour M, Dempsey AA, Urowitz MB, et al. Association of a gene expression profile from whole blood with disease activity in systemic lupus erythematosus. Ann Rheum Dis 2008;67:1069-75.

38. Kong KO, Tan AW, Thong BY, et al. Enhanced expression of interferon-inducible protein-10 correlates with disease activity and clinical manifestations in systemic lupus erythematosus. Clin Exp Immunol 2009;156:134-40.

39. Landolt-Marticorena C, Bonventi G, Lubovich A, et al. Lack of association between the interferon-alpha signature and longitudinal changes in disease activity in systemic lupus erythematosus. Ann Rheum Dis 2009;68:1440-6. 